\title{
CONCEPÇÕES TEÓRICAS E METODOLÓGICAS DO CUIDADO TRANSDISCIPLINAR: UMA REVISÃO INTEGRATIVA
}

Cristiano Bertolossi Marta', Enéas Rangel Teixeira² ${ }^{2}$ Márcio Tadeu Ribeiro Francisco ${ }^{3}$, Carlos Eduardo Nunes Ferreira ${ }^{4}$, Priscilla Oliveira da Silva ${ }^{5}$, Carlos Roberto Lyra da Silva ${ }^{6}$, Tatiana Araújo de Lima ${ }^{7}$, Patrícia Xavier Hommerding Frasson ${ }^{8}$, Monyque Evelyn dos Santos Silva ${ }^{9}$, Otto Guilherme Gerstenberger Júnior ${ }^{10}$, Raphaela Nunes Alves $^{11}$, Roberto Carlos Lyra da Silva ${ }^{12}$, Pedro Ruiz Barbosa Nassar ${ }^{13}$

\section{Resumo}

Introdução: Objetivou-se realizar um levantamento da produção científica na enfermagem e suas interfaces sobre as concepções teóricas e metodológicas do cuidado e discutir o cuidado na enfermagem e suas interfaces na perspectiva da complexidade em atitude transdisciplinar. Métodos: Trata-se de uma revisão integrativa que utilizou o Portal de Periódicos da CAPES, no período entre 2005 e 2015. A amostra foi composta por 21 trabalhos de pesquisas. Definiu-se a categoria: As concepções teóricas e metodológicas utilizadas na Enfermagem e suas interfaces para discutir o cuidado em atitude transdisciplinar. Resultados: As pesquisas objetivaram compreender e aplicar a complexidade do cuidado baseadas em conhecimentos sobre a complexidade, a importância com o ambiente e sobre as influências do cotidiano na determinação do cuidado a ser prestado. Conclusão: O estudo mostrou que há convergência entre os discursos e metodologias utilizados pelos autores corroborando com as ideias defendidas pela complexidade.

Descritores: Cuidados de Enfermagem, Conceitos de Cuidar, Pensamento Complexo

1 Professor do Curso de Graduação e Pós-Graduação em Enfermagem e Diretor de Pesquisa da Universidade Veiga de Almeida e Professor Adjunto do Departamento de Fundamentos de Enfermagem da Faculdade de Enfermagem da Universidade do Estado do Rio de Janeiro.

${ }^{2}$ Titular da Escola de Enfermagem Aurora Afonso Costa da Universidade Federal Fluminense.

${ }^{3}$ Professor Titular do Curso de Graduação em Enfermagem da Universidade Veiga de Almeida e Livre Docente e Professor Adjunto da Faculdade de Enfermagem da Universidade do Estado do Rio de Janeiro.

${ }^{4}$ Pró-reitor de Graduação e Professor do Curso de Graduação em Arquitetura e Urbanismo da Universidade Veiga de Almeida.

${ }_{5}^{5}$ Professora do Curso de Graduação em Enfermagem da Universidade Veiga de Almeida e Doutoranda em Saúde Pública da Escola Nacional de Saúde Pública da Fundação Oswaldo Cruz (ENSP-FIOCRUZ).

${ }^{6}$ Professor Associado da Escola de Enfermagem Alfredo Pinto e Diretor de Pós-Graduação da Universidade Federal do Estado do Rio de Janeiro.

${ }^{7}$ Professora do Curso de Graduação em Odontologia e Fonoaudiologia da Universidade Veiga de Almeida e Professora Adjunta da Faculdade de Odontologia da Universidade do Estado do Rio de Janeiro.

${ }^{8}$ Professora do Curso de Graduação em Fisioterapia da Universidade Veiga de Almeida e Pós-doutoranda da Universidade Federal do Estado do Rio de Janeiro.

${ }^{9}$ Professora do Curso de Graduação em Enfermagem da Universidade Veiga de Almeida.

${ }^{10}$ Doutorando do Programa de Pós-Graduação em Psicanálise, Saúde e Sociedade da Universidade Veiga de Almeida.

${ }^{11}$ Professora do Curso de Graduação em Enfermagem da Universidade Veiga de Almeida e Professora Adjunta do Departamento de Fundamentos

de Enfermagem da Faculdade de Enfermagem da Universidade do Estado do Rio de Janeiro.

${ }_{12}$ Associado da Escola de Enfermagem Alfredo Pinto da Universidade Federal do Estado do Rio de Janeiro.

${ }^{13}$ Professor do Curso de Graduação em Enfermagem da Universidade Veiga de Almeida. 


\section{THEORETICAL AND METHODOLOGICAL CONCEPTIONS OF TRANSDISCIPLINARY CARE: AN INTEGRATIVE REVIEW}

Cristiano Bertolossi Marta, Enéas Rangel Teixeira, Márcio Tadeu Ribeiro Francisco, Carlos Eduardo Nunes Ferreira, Priscilla Oliveira da Silva, Carlos Roberto Lyra da Silva, Tatiana Araújo de Lima, Patrícia Xavier Hommerding Frasson, Monyque Evelyn dos Santos Silva, Otto Guilherme Gerstenberger Júnior, Raphaela Nunes Alves, Roberto Carlos Lyra da Silva, Pedro Ruiz Barbosa Nassar

\section{Abstract}

Introduction: The purpose of this study was to conduct a survey of the scientific production in nursing and its interfaces on the theoretical and methodological conceptions of care and to discuss care in nursing and its interfaces in the perspective of complexity in a transdisciplinary attitude. Methods: This is an integrative review that used the Portal of Periodicals of CAPES, in the period between 2005 and 2015. The sample consisted of 21 research papers. The category was defined: The theoretical and methodological conceptions used in Nursing and its interfaces to discuss care in a transdisciplinary attitude. Results: The research aimed to understand and apply the complexity of care based on knowledge about complexity, importance with the environment and on the influences of daily life in determining the care to be provided. Conclusion: The study showed that there is convergence between the discourses and methodologies used by the authors corroborating with the ideas defended by the complexity. 


\section{CONCEPTOS TEÓRICOS Y METODOLÓGICOS DEL CUIDADO TRANSDISCIPLINARIO: UNA REVISIÓN INTEGRATIVA}

Cristiano Bertolossi Marta, Enéas Rangel Teixeira, Márcio Tadeu Ribeiro Francisco, Carlos Eduardo Nunes Ferreira, Priscilla Oliveira da Silva, Carlos Roberto Lyra da Silva, Tatiana Araújo de Lima, Patrícia Xavier Hommerding Frasson, Monyque Evelyn dos Santos Silva, Otto Guilherme Gerstenberger Júnior, Raphaela Nunes Alves, Roberto Carlos Lyra da Silva, Pedro Ruiz Barbosa Nassar

\section{Resumen}

Introducción: El objetivo del trabajo era realizar un levantamiento de la producción científica en la enfermería y sus interfaces sobre las concepciones teóricas y metodológicas del cuidado y discutir el cuidado en la enfermería y sus interfaces en la perspectiva de la complejidad en actitud transdisciplinaria. Métodos: Se trata de una revisión integrativa que utilizó el Portal de Periódicos de la CAPES, en el periodo entre 2005 y 2015. La muestra fue compuesta por 21 trabajos de investigación. Se definió la categoría: Las concepciones teóricas y metodológicas utilizadas en la Enfermería y sus interfaces para discutir el cuidado en actitud transdisciplinaria. Resultados: Las investigaciones objetivaron comprender y aplicar la complejidad del cuidado basadas en conocimientos sobre la complejidad, la importancia con el ambiente y sobre las influencias de lo cotidiano en la determinación del cuidado a ser prestado. Conclusión: El estudio mostró que hay convergencia entre los discursos y metodologías utilizados por los autores corroborando con las ideas defendidas por la complejidad. 


\section{Introdução}

O cuidado tem se tornado um tema de destaque na enfermagem, na saúde e nas ciências humanas. $\mathrm{O}$ interesse por esse tema na profissão reflete-se pelo número crescente de estudos encontrados na literatura nacional e internacional expressando-se também, através da realização de eventos e na criação de grupos de pesquisas, que tem como temática central o cuidado. A complexidade do cuidar parece estar cada vez mais evidente no ser e fazer do enfermeiro, levando-o à busca de uma prática que redireciona a sua competência como um profissional, envolvido com outros seres, situando-se no ambiente do cuidado, no cenário de múltiplas interações humanas (1).

O fenômeno do cuidado não é somente uma experiência subjetiva e material. Ele é também historicamente construído, sendo que, circunstâncias particulares, ideologias e relações de poder criam as condições sob as quais o cuidado pode ocorrer, as formas que assumirá e as consequências que terá sobre aqueles que o realizam e o recebem. O cuidado é considerado função primordial na sobrevivência de todo ser vivo, especialmente do ser humano.

O cuidar é mais que um ato; é uma atitude de ocupação, preocupação, responsabilização e de desenvolvimento afetivo com o outro. A atitude de cuidar gera atos que denotam preocupação com as pessoas, zelo pelas relações de amizade, interesse pelo bem-estar, desvelo para tornar o ambiente agradável e deligência para resolver os assuntos. Ressalta-se que o ser

humano é único, livre e criativo, e como ele desenvolve sua habilidade de cuidar de si mesmo, dos outros, do planeta, tem interesse em detectar e decidir sobre o sentido de cuidar (2).

Na Enfermagem, o cuidado começou a trilhar caminhos distintos desde a sua origem. Porém, foi marcadamente através de Florence Nightingale que, dada a sua vocação para o cuidar, mostrou o exercício do cuidado dentro de uma visão holística do ser humano e valorizou os fatores ambientais, entendendo como influentes no comportamento humano. O modelo nightingaleano difundiu-se e influenciou, por anos, os cuidados realizados pela Enfermagem em muitos países, inclusive no Brasil. Posteriormente, outros referenciais emergiram e se consolidaram, colocando o cuidado como essência e/ou objeto central da Enfermagem (3).

No seu dia-a-dia, os profissionais de enfermagem utilizam a ciência, a arte e a ética no processo de promoção, manutenção e recuperação da saúde, por meio de ações de cuidado destinadas a ajudar as pessoas a viverem mais saudáveis e, quando preciso, a superarem os efeitos da doença como um fenômeno social, existencial, cultural e transitório. O cuidado pensando autenticamente como existencial, essencial do ser humano, diz respeito a zelo, desvelo, atenção, bom trato e solicitude, constituindo-se, dessa forma, um fundante por meio do qual a pessoa sai de si para se centrar na preocupação com o outro. Para o autor, o cuidado é o caráter existencial mais próximo do ser humano (4).

A partir do final da década de 1970, com maior ênfase na última década do século passado, o cuidado vem sendo discutido 
e tornou-se foco de muitas pesquisas realizadas por enfermeiros. Na década de 1990 passou a ser o marco referencial em um momento de mudanças significativas na enfermagem, com vistas a uma nova forma de pensar-fazer-ensinar-aprender enfermagem.

O cuidado é indispensável ao ser humano, sendo a essência da prática de Enfermagem e função precípua do enfermeiro. Há de ser conceituado e compreendido por todos, como os que oferecem cuidados (enfermeiras assistenciais), para quem recebe os cuidados (paciente/cliente), para educadores e educandos da área de Enfermagem, como o catalisador do fazer da enfermagem (5).

Entre as necessidades de desenvolvimento de conceitos de enfermagem, o processo de fazer-ensinar requer a compreensão do conceito de cuidado e do processo de cuidar em enfermagem. Desse modo, o fazer e o ensinar poderão ter significado para todos os envolvidos neste processo -enfermeiros e alunos de enfermageme, ainda, possibilitarão a construção de outros conceitos que atendem ao processo fazer-ensinar enfermagem nos diversos cenários da prática de enfermagem.

No processo de saber-fazer-ensinar enfermagem, com ênfase no cuidado nos cenários da prática hospitalar, na saúde pública e na academia, nossa grande preocupação sempre esteve e, ainda permanece, no ato de cuidar e na forma como este é ensinado nos cursos de graduação em enfermagem. Diante do exposto este estudo possui a seguinte questão norteadora:

\section{0 que se tem produzido na literatura científica da enfermagem e da área da saúde sobre conceitos de cuidado?}

Para responder essa questão definiu-se como objetivos:

1. Realizar um levantamento da produção científica na enfermagem e suas interfaces sobre as concepções teóricas e metodológicas do cuidado.

2. Discutir o cuidado na enfermagem e suas interfaces na perspectiva da complexidade em atitude transdisciplinar.

\section{Justificativa}

O cuidado de enfermagem é um conceito teórico e filosófico, que subsidia estudos e pesquisas e norteia a prestação de serviço na saúde. O cuidar mantém a vida e garante a satisfação de um conjunto de necessidades indispensáveis ao sujeito. As formas de cuidado são diversificadas na sua manifestação de acordo com o contexto sociocultural. Essa pesquisa contribui para a área de conhecimento de enfermagem e suas interfaces na perspectiva da inter e a transdisciplinaridade. Por conseguinte, enfoca-se o cuidado na enfermagem e suas interfaces.

Ao aprofundar a pesquisa e encontrar as concepções teóricas e metodológicas utilizadas nesse contexto tornou-se possível identificar a possibilidade de interseção entre as profissões que compõem a área da saúde.

O estudo visa fortalecer reflexões e pesquisas sobre a complexidade do cuidado 
em enfermagem e suas interfaces. Trata-se de uma temática emergente, da qual é necessário novas formas de saber e fazer, atendendo a especificidade da área de conhecimentos e suas conexões para consubstanciar ações de transformação das práticas de saúde no Brasil.

A busca e exposição dos dados e artigos já existentes trarão benefícios para o ensino, pois promoverá discussões sobre os caminhos percorridos com as concepções utilizadas e as contribuições que elas poderão oferecer para os futuros profissionais no desempenho de suas profissões.

O conhecimento dessas concepções de cuidado auxiliará a ampliação das discussões na interdisciplinaridade e na transdisciplinaridade. $\mathrm{Na}$ interdisciplinaridade, ocorre troca de conhecimentos, interação e dialogicidade. $-\mathrm{A}$ interdisciplinaridade tem uma ambição diferente daquela da pluridisciplinaridade. Ela diz respeito à transferência de métodos de uma disciplina para outra- $(6: 82)$.

Já a transdisciplinaridade -é uma evolução qualitativa, alicerçada no método sintético, enfatizando a totalidade real, apresentando, portanto, uma postura ampliativa" (6:83). A transdisciplinaridade permite maior conexão e abertura através das disciplinas, problematiza as dicotomias e divisões binárias. A transdisciplinaridade faz parte da cisão complexa do mundo e do cosmos. Tanto a interdisciplinaridade quanto a transdisciplinaridade não são correntes teóricas ou filosóficas, mas atitudes e depende da disposição e decisões políticas, pessoais, grupais, intersubjetivas

\section{Materiais e métodos}

O trabalho trata de uma revisão integrativa, cujo substrato é o cuidado em enfermagem e suas interfaces. Assim, busca-se delinear sobre o ponto de vista conceitual, a complexidade do cuidado e suas interfaces com a enfermagem e saúde em uma atitude transdisciplinar.

Desse modo, o estudo pretende responder a pergunta: o que se tem produzido na literatura científica da enfermagem e suas interfaces sobre conceitos de cuidado. Para tanto, foram adotadas como fonte de informação as bases eletrônicas de dados o Portal de Periódicos da CAPES, no período entre 2005 e 2015.

A coleta de dados ocorreu em 2013. Foi realizado cruzamento binômio com os unitermos: cuidados de enfermagem $x$ conceitos de cuidar x pensamento complexo.

Os critérios estabelecidos para a inclusão dos trabalhos foram: periódicos e artigos publicados entre os anos de 2005 e 2015 , procedência nacional, idioma português e que tenha uma abrangência sobre o cuidado de forma geral. Foram excluídas todas as publicações que não atenderem aos critérios de inclusão descritos.

Para analisar os artigos encontrados, realizou-se primeiramente a leitura sistemática dos textos. Posteriormente, foi verificada a relação entre os resultados das publicações de modo que estas permitissem identificar os conceitos de cuidados utilizados na enfermagem e na área da saúde e as concepções teóricas e metodológicas que as subsidiam. 
Após a análise, os dados foram transcritos e alocados em uma tabela para melhor exposição dos resultados e discussões.

\section{Resultados}

A partir das buscas nas bases de dados científicas, inicialmente 75 publicações representaram o universo pesquisado com forte potencial de inclusão na revisão integrativa, pois abordavam questionamentos, conceitos e reflexões a cerca do trabalho de profissionais da equipe de saúde.

Após a leitura dos resumos e análise dos trabalhos científicos, esse número foi reduzido, tendo em vista que o presente estudo baseia-se em critérios de inclusão e exclusão bem definidos, quais sejam: ter uma abrangência sobre o cuidado de forma geral em seu contexto e que permita a identificação das concepções teóricas e metodológicas utilizadas para seu desenvolvimento.

Uma vez aplicado os critérios de inclusão, esse número foi redimensionado para 21 estudos compondo a amostra total a ser discutida nesse trabalho.

Quanto à distribuição dos artigos incluídos no estudo de acordo com os critérios estabelecidos e o ano de publicação, foram identificados 1 artigo no volume de 2015, 1 artigo em 2014, 2 em 2013, 2 artigos em 2012, 1 em 2011, 2 em 2010, 3 em 2009, 1 pesquisa em 2008 e 2007, 3 artigos em 2006, 1 trabalho de pesquisa em 2005, 2 em 2004 e 1 em 2002.
Após a seleção dos artigos, encontramos as seguintes concepções teóricas: 7 artigos baseados no referencial de complexidade segundo Edgar Morin; 12 abordaram a construção do conceito de cuidado de enfermagem, interdisciplinaridade e/ ou equipe multiprofissional ressaltando a importância com o ambiente e com a organização dos cuidados de Enfermagem de Florence Nightingale e as necessidades humanas básicas de Wanda Aguiar Horta; e 2 sobre as influencias do cotidiano de Michel Certeau na determinação do cuidado a ser prestado.

Quanto às concepções metodológicas e tipo de estudo, destacam-se 11 trabalhos de pesquisa de campo ( 7 de Representação Social (RS), 2 de Teoria Fundamentada nos Dados (TFD), 1 de Abordagem Multirreferencial sobre o cuidado e 1 de Epistemologia da complexidade), 8 de Reflexão crítica, 1 de Revisão sistemática e 1 de Revisão integrativa (figura 1).

Federal, 2 em Florianópolis, 1 em Goiás. A origem das publicações foi diversificada entre as regiões do Brasil, percebendo-se assim um predomínio de publicações na região sudeste (13 artigos).

Os resultados levaram a construção da seguinte categoria: As concepções teóricas e metodológicas utilizadas na Enfermagem e suas interfaces para discutir o cuidado em atitude transdisciplinar.

Por meio desta revisão integrativa evidencia-se que os artigos foram publicados: 5 no Rio de Janeiro, 8 em São Paulo, 1 no Paraná, 4 no Distrito 


\begin{tabular}{|c|c|c|c|c|c|c|c|c|c|c|c|c|}
\hline 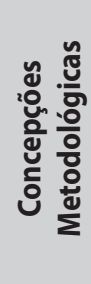 & 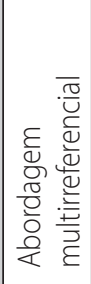 & 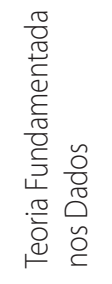 & 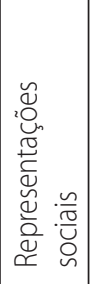 & 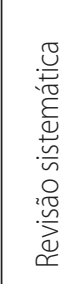 & 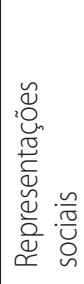 & 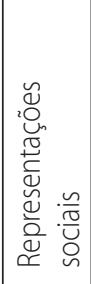 & 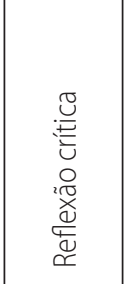 & 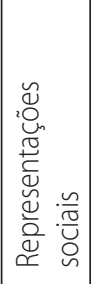 & 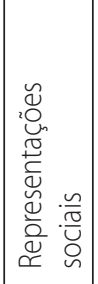 & 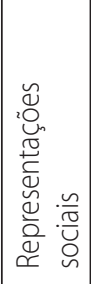 & 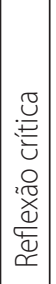 & 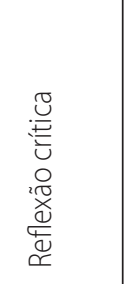 \\
\hline 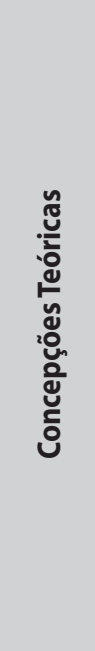 & 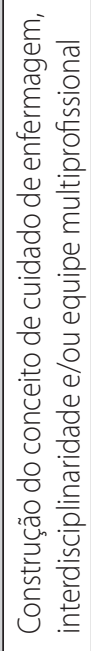 & 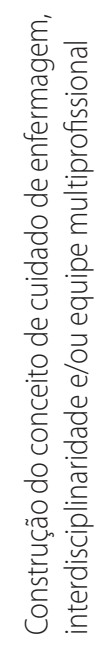 & 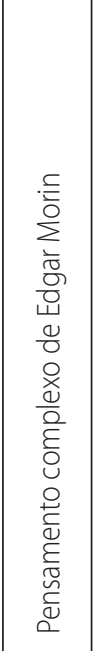 & 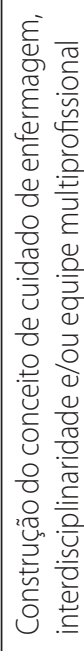 & 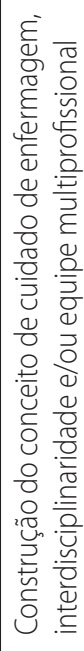 & 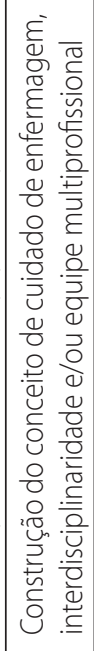 & 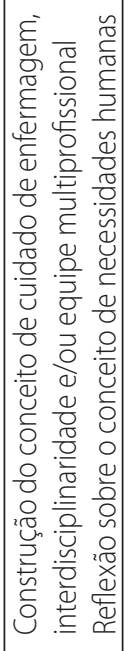 & 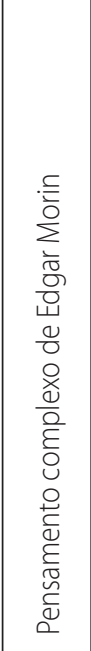 & 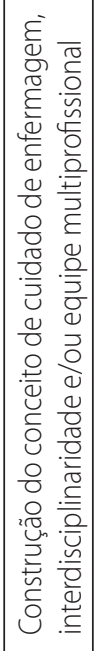 & 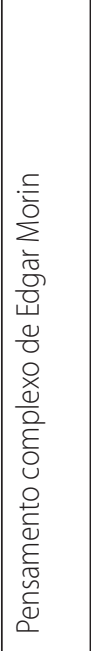 & 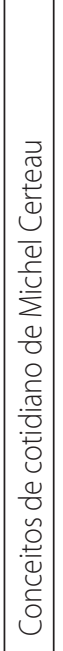 & 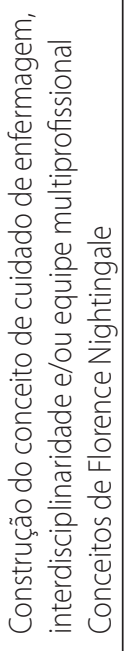 \\
\hline 을 & 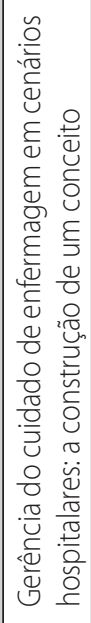 & 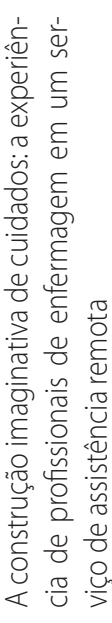 & 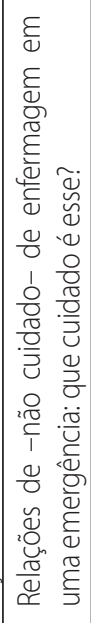 & 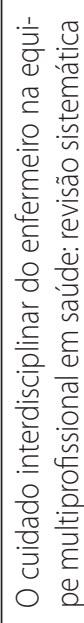 & 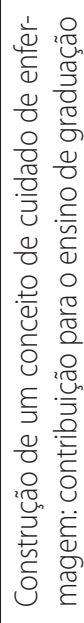 & 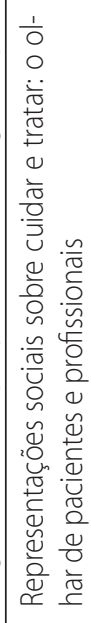 & 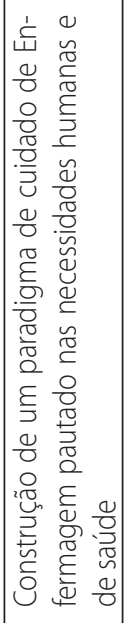 & 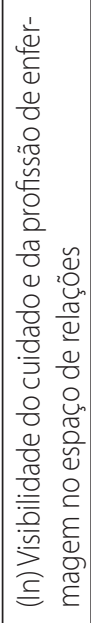 & 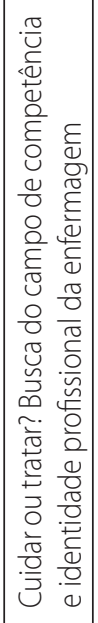 & 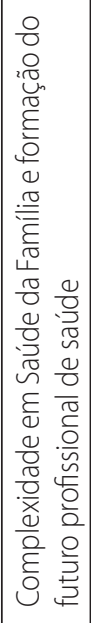 & 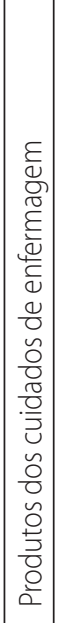 & 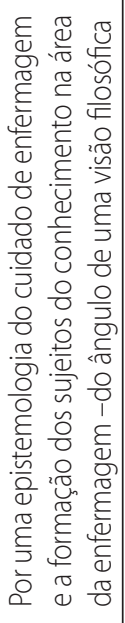 \\
\hline 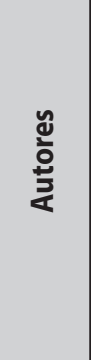 & 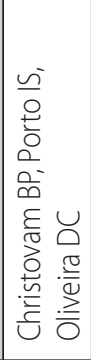 & 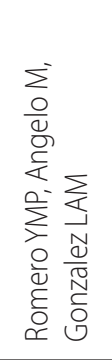 & 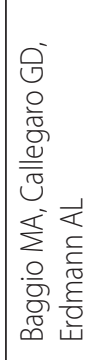 & 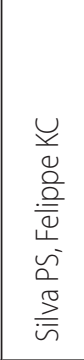 & 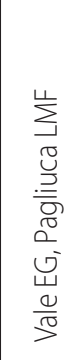 & 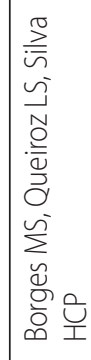 & 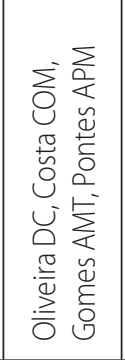 & 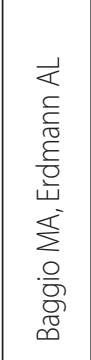 & 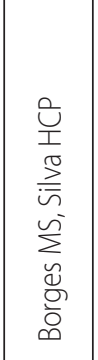 & 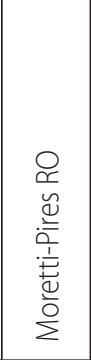 & $\left|\begin{array}{l}\bar{z} \\
0 \\
\frac{0}{\bar{Q}} \\
\circlearrowleft\end{array}\right|$ & 常 \\
\hline 总 巴 & $\bar{~}$ & & $\ddot{\Psi}$ & 岀 & 岃 & 过 & ذ & 叫 & ш & $\bar{\Psi}$ & ш & \\
\hline
\end{tabular}




\begin{tabular}{|c|c|c|c|c|c|c|c|c|c|}
\hline 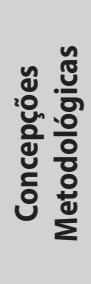 & 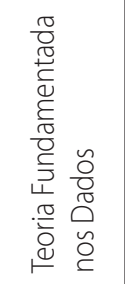 & 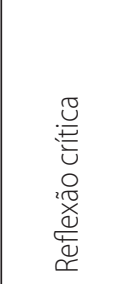 & 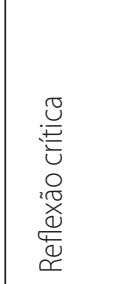 & 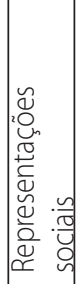 & 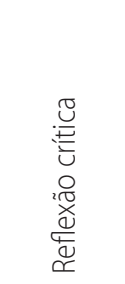 & 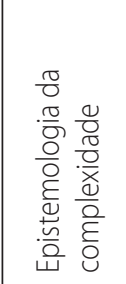 & 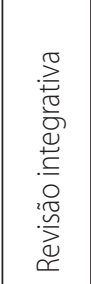 & 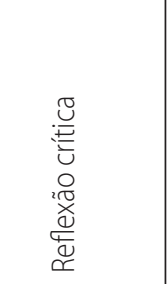 & 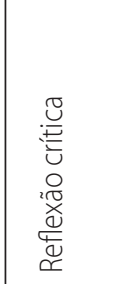 \\
\hline 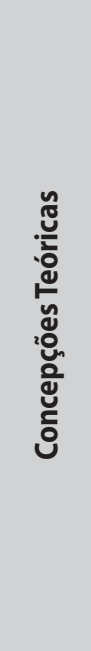 & 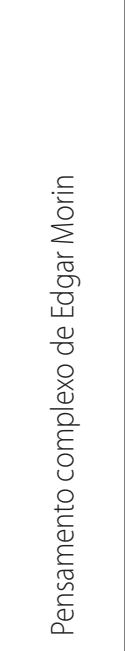 & 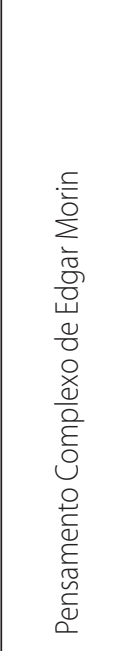 & 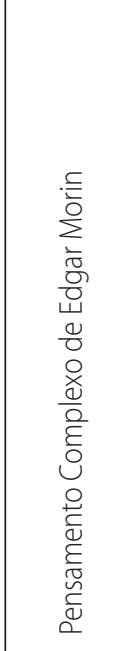 & 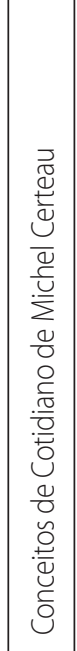 & 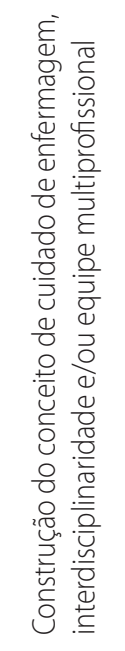 & 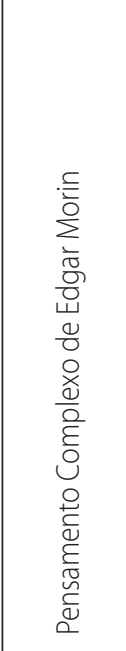 & 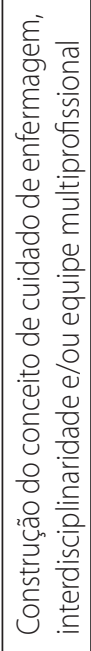 & 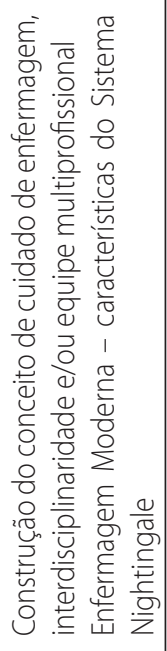 & 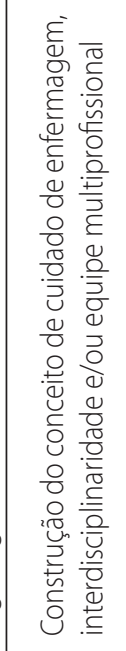 \\
\hline 元 & 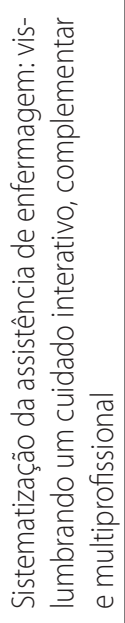 & 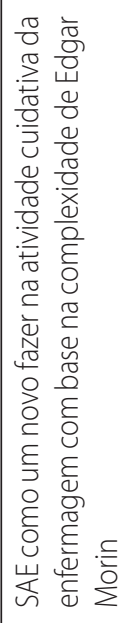 & 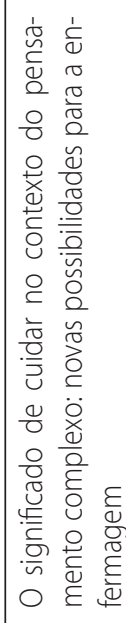 & 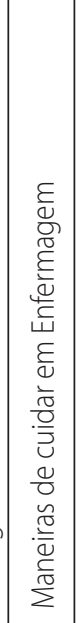 & 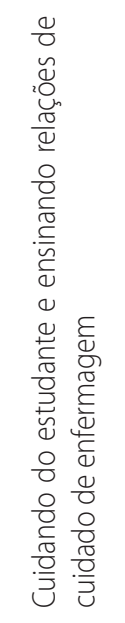 & 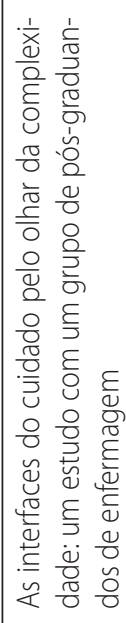 & 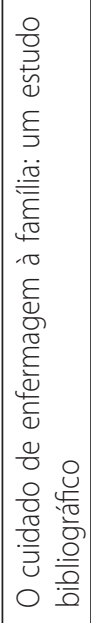 & 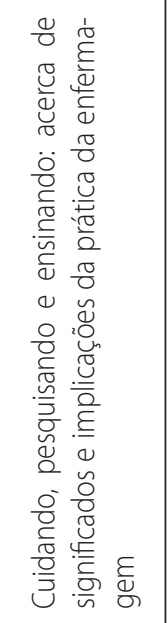 & 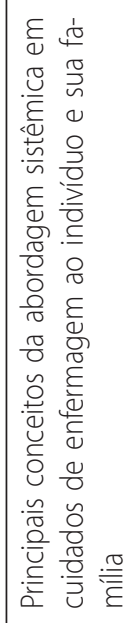 \\
\hline 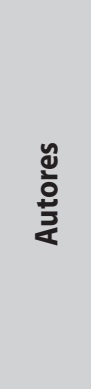 & 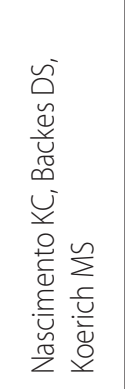 & 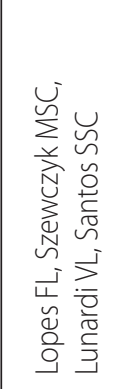 & 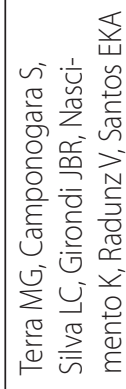 & $\begin{array}{l}\sum \\
\circ \\
\overline{\bar{D}} \\
\dot{\cup}\end{array}$ & 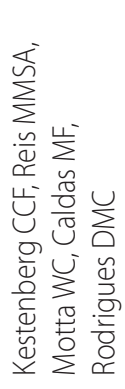 & 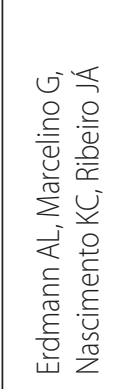 & 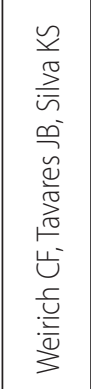 & $\frac{\frac{c}{\sqrt{5}}}{2}$ & 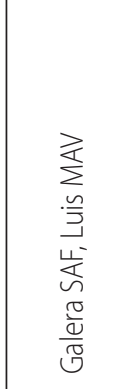 \\
\hline 递 & $\bar{\square}$ & & $\bar{\square}$ & $\bar{w}$ & $\overline{ }$ & $\bar{\square}$ & $\bar{\square}$ & ய & ن \\
\hline
\end{tabular}




\section{Discussão}

A leitura na íntegra dos artigos desta revisão permitiu a visualização de determinadas concepções teóricas convergentes que possibilitaram o agrupamento dos artigos por similaridade de conteúdo. Percebemos a menção de artigos sobre o pensamento complexo de Edgar Morin; os conceitos de interdisciplinaridade e/ou equipe multiprofissional para construção de conceitos de cuidar; e sobre conceitos de Cotidiano de Michel Certeau utilizados por Coelho nas definições dos tipos de cuidar. Junto a isso, percebemos a utilização de concepções metodológicas como: Representação Social, Teoria Fundamentada nos Dados (TFD), Abordagem Multirreferencial sobre o cuidado, Epistemologia da complexidade, Reflexão crítica, Revisão Sistemática e Revisão Integrativa. A relação entre essas concepções teóricas e metodológicas foi descrita através de uma categoria intitulada: As concepções teóricas e metodológicas utilizadas na Enfermagem e suas interfaces para discutir o cuidado em atitude transdisciplinar que veremos a seguir.

\section{As concepções teóricas e metodológi- cas utilizadas na Enfermagem e suas interfaces para discutir o cuidado em atitude transdisciplinar}

Na área da saúde, as maneiras de cuidar do cliente proporcionam a criação de produtos e um conjunto de saberes, pois, ao criar, há uma construção de saberes tecidos a partir de distintas e integradas contribuições. Por conseguinte, esses conhecimentos partem do conjunto de contribuições da sociologia, química, antropologia, enfermagem, medicina, serviço social, psicologia, nutrição, entre outras e de contradições da vida cotidiana.

A maioria dos textos encontrados na busca realizada corrobora com essa afirmativa supracitada e versa sobre interdisciplinaridade e/ou equipe multidisciplinar na construção de um conceito de cuidado baseado nas concepções de Enfermagem e suas interfaces teóricas que fundamentam as práticas assistenciais como: a influência do ambiente na saúde defendida por Florence Nightingale, processo de enfermagem de Wanda Aguiar Horta e o cotidiano que influencia e determina o tipo de cuidado necessário a cada indivíduo de Michel Certeau.

Concomitante às concepções teóricas explicitadas, abordamos as concepções metodológicas encontradas em nossa busca e a relação entre elas, de modo a enfatizar as convergências e divergências existentes nessa união.

É importante ressaltar que a metodologia é o conjunto de estratégias para coletar informações acerca da realidade examinada pelo pesquisador e no contexto da realização de uma pesquisa empírica. Já a concepção teórica é o conjunto de pensamentos, ideias e opiniões sobre determinado assunto defendido por um teórico ou grupo de teóricos.

Partindo dessa afirmativa, apresentamos as concepções teóricas e metodológicas utilizadas nas pesquisas presentes nesse levantamento.

O cuidado é uma das mais antigas práticas da história do mundo, cuja função é assegurar a continuidade da vida do 
grupo e da espécie, tendo em vista a garantia das funções vitais. Por muito tempo, teve um caráter apenas de tradição cultural, transmitido através da experiência passada de geração a geração (8). No entanto, aos poucos passou por um processo de profissionalização, sendo um atributo de profissionais de saúde, porém ganhou espaço e singularidade na busca da profissionalização da enfermagem. Obviamente, esta profissionalização foi marcada por inúmeros fatos históricos, mas é possível evidenciar, na Enfermagem, uma forte herança advinda de um vínculo ligado à moralidade e religiosidade (9).

Na Enfermagem, o cuidado significa um trabalho profissional específico. O termo -cuidado-, na Enfermagem, denota zelo pelo bem-estar ou pela saúde, preocupação, interesse por alguém, além de indicar ações dinâmicas e interrelacionadas. Portanto, o cuidar/cuidado designa uma atividade que é merecedora de reflexões e possibilidades de mudanças, de transformações, de interações e do entendimento de sua complexidade (10).

Assim, a Enfermagem foi, historicamente, influenciada por fatores variados, desenvolvendo-se ao ritmo das grandes correntes de pensamento que marcaram a evolução do conhecimento. A prática da enfermagem, na primeira metade do século XX, vai se distanciando dos valores morais e religiosos herdados do passado e adota o modelo biomédico, valorizando o tecnicismo. Dessa maneira, a Enfermagem relegou, a segundo plano, um cuidado que tenha como sentido o de assegurar a continuidade da vida dos homens e a sua razão de existir, criando-se um imenso abismo.
Com disso, a enfermagem, ao adotar durante anos este modelo, distanciou-se de sua origem (8:68).

A função precípua do enfermeiro é o cuidado de enfermagem, cujo objetivo centra-se na promoção da saúde, na prevenção de doenças e na recuperação e reabilitação da saúde. Como toda a ciência, a enfermagem carece consolidar um corpo de conhecimentos próprios e uma linguagem específica que permitam aos seus exercentes compreender seu fazer e, assim, prestar cuidados significativos capazes de atender às reais necessidades dos seres humanos por eles assistidos $(5,22)$.

Nesses termos, a concepção de Florence Nightingale condiz com ideias e conceitos de ações do cuidado, tão consistente com princípios básicos aos atos dos enfermeiros. Os quais são inerentes aos princípios, aos enunciados do modelo de ensino e de treinamento adotados para formar uma nova mentalidade profissional consoante aos significados da arte da enfermagem e com o cuidado prestado pelos enfermeiros.

Os estudos, que utilizaram como concepção teórica o conceito Florence Nightingale sobre a influência do ambiente na saúde do indivíduo, adotaram três tipos de caminho metodológico: a reflexão crítica, a abordagem multirreferencial e revisão integrativa.

O casamento com cada uma dessas metodologias resultou numa contribuição importante, visto a influência na forma de olhar para o tema. Diante disso, em um dos textos de Carvalho (2004) foi discutido o conteúdo por meio da reflexão 
crítica, que procurou pensar questões que implicam as ideias acerca da Enfermagem, seu saber e sua história, enfatizando que a epistemologia do cuidado em Enfermagem precisa referenciar às dimensões teóricas, filosóficas e históricas relativas à própria Enfermagem.

Como a pesquisa de reflexão crítica é uma tomada de consciência, a autora $(12,13)$ se propôs a examinar ou analisar fundamentos do cuidado de Enfermagem. Refletir criticamente é a atitude de investigar e para isso é necessário conhecer aquilo que é investigado, sem nenhum tipo de preconceitos e pré-conceitos. Refletir criticamentetambém é posicionar-se a partir de um conjunto de informações conquistados com a pesquisa. E é justamente isso o que a autora faz quando reconstrói e/ou revive os aspectos históricos da profissão abordando desde seu nascimento até a formação dos sujeitos do conhecimento na área da enfermagem.

A convergência entre a teoria e a metodologia permitiu um enriquecimento do conteúdo discutido, o que nos proporcionou uma discussão mais aprofundada. Essa mesma conexão positiva também ocorreu no estudo com a mesma teoria, porém com o uso da revisão integrativa que é um dos métodos de pesquisa utilizados na Pesquisa Baseada em Evidências (PBE) que permite a incorporação das evidências na prática clínica. Esse método tem a finalidade de reunir e sintetizar resultados de pesquisas sobre um delimitado tema ou questão, de maneira sistemática e ordenada, contribuindo para o aprofundamento do conhecimento do tema investigado. Desde 1980, a revisão integrativa é relatada na literatura como método de pesquisa (14).
Junto a isso, a revisão integrativa inclui a análise de pesquisas relevantes que dão suporte para a tomada de decisão e a melhoria da prática clínica, possibilitando a síntese do estado do conhecimento de um determinado assunto, além de apontar lacunas do conhecimento que precisam ser preenchidas com a realização de novos estudos. Este método de pesquisa permite a síntese de múltiplos estudos publicados e possibilita conclusões gerais a respeito de uma particular área de estudo. É um método valioso para a enfermagem, pois muitas vezes os profissionais não têm tempo para realizar a leitura de todo o conhecimento científico disponível devido ao volume alto, além da dificuldade para realizar a análise crítica dos estudos.

Outra concepção metodológica também utilizada para abordar sobre os conceitos de Florence Nightingale foi a abordagem multirreferencial. Nesse sentido:

a análise multirreferencial das situações das práticas dos fenômenos e dos fatos educativos se propõe explicitamente uma leitura plural de tais objetos, sob diferentes ângulos e em função de sistemas de referencias distintos, os quais não podem reduzir-se uns aos outros. Muito mais que uma posição metodológica, trata-se de uma decisão epistemológica. (15:7)

A pesquisa que versou sobre a construção de um conceito de gerência em Enfermagem, se deparou com um extenso corpo de informações provenientes de um olhar fundamentado na análise multirreferencial. Esse olhar estimulou a construção de um conceito que permitiu um equilíbrio entre o cuidar e o administrar através da mudança na formação 
e nos saberes e fazeres dos enfermeiros na organização da assistência de enfermagem em diferentes cenários, de modo que os padrões de prestação de cuidados de enfermagem implementados por elas na prática sejam fundamentados em um modelo sistêmico.

Como vimos, a concepção teórica adequou-se as três concepções metodológicas. Embora, elas apresentem afinidades e práticas aproximadas, existem peculiaridades importantes de serem mencionadas.

A Reflexão crítica exige um aprofundamento maior por parte do pesquisador, além de exigir um conhecimento apropriado sobre a temática. Tal cobrança não aparece na revisão integrativa e na abordagem multirreferencial.

Revisão integrativa é um método de pesquisa que envolve a sistematização e publicação dos resultados de uma pesquisa bibliográfica em saúde para que possam ser úteis na assistência à saúde, acentuando a importância da pesquisa acadêmica na prática clínica. $\mathrm{O}$ principal objetivo da revisão integrativa é a integração entre a pesquisa científica e a prática profissional no âmbito da atuação profissional.

Já a abordagem multirreferencial se caracteriza pelo olhar diferenciado e diversificado sobre a mesma pesquisa, o que permite uma ampliação e diferenciação da opinião sobre o mesmo objeto.

Ressalta-se também a importância da enfermagem como uma ciência que busca, através da pesquisa, a realidade e a atualidade dos fatos e dos procedimentos possibilitando a execução de um cuidado mais seguro e fidedigno com as necessidades do indivíduo.

Dentro desse contexto de pesquisa e modelo para atuação profissional, configura-se como protagonista Wanda Aguiar Horta, que adaptou a Teoria das Necessidades Humanas de Abraham Maslow à Enfermagem, dando forma aos nossos conceitos e criando o Processo de Enfermagem contendo 6 etapas: Histórico de Enfermagem (Anamnese e Exame Físico), Diagnóstico de Enfermagem, Plano de Assistência de Enfermagem, Plano de Cuidados de Enfermagem, Evolução de Enfermagem e Prognóstico de Enfermagem (16).

O propósito do trabalho de pesquisa que relata Wanda Aguiar Horta era de refletir sobre o conceito de necessidades humanas e de saúde e sua pertinência ao cuidado de enfermagem/de saúde, a partir de uma reflexão crítica.

Nessa perspectiva, o cuidado de enfermagem e as suas interfaces, abordado pelo referido trabalho científico, ganham sentido e contexto como ações intencionais, institucionalmente localizadas e socialmente determinadas. Enquanto forma particular de agir no mundo, o cuidado de enfermagem é constituído por um saber e por seus desdobramentos materiais e não materiais, inseridos na produção de serviços de saúde.

São nas interações sociais diárias que se constroem mutuamente tanto o objeto do trabalho (cuidado) quanto à representação do profissional (identidade). Então, será a partir de novas interpretações sobre uma nova maneira de cuidar que se poderão transformar as crenças e os valores frente 
aos diferentes grupos que interagem no espaço profissional da assistência à saúde, resultando no reconhecimento do valor social da enfermagem.

Para subsidiar essa reflexão teórica cotidiana de cuidados como produtos ou produtos como cuidados dentro e fora das disciplinas ou nas conversas informais nos hospitais em especial, é importante ressaltar a existência dos conceitos de Cotidiano de Certeau, que diz -extrair do seu ruído (cliente) as maneiras de fazer"17:921; de cuidar, como processo de expressão, de reflexão, de elaboração do pensamento, de imaginação, de meditação e de aplicação intelectual [...]; O cuidado é visto como uma -ação imediata prestada [...], em curto espaço de tempo, desenvolvido em vários momentos, envolvendo segurança e competência, aliadas à tecnologia específica que a situação exige" (17:921).

A partir desse pensamento, conseguimos visualizar a necessidade de uma organização para a prestação de cuidados a uma pessoa, como por exemplo, o processo de enfermagem para um determinado cliente. Essa prática é um planejamento estratégico da profissão enfermagem para realização de suas funções, algo que certamente as demais profissões de uma equipe multidisciplinar também possuem. Com isso, entende-se que o cotidiano definido por Michel de Certeau, traz uma proposta de uma inversão de perspectiva, de um deslocamento da atenção: dos produtos recebidos para a criação anônima. Encontrar sentidos nas artes de fazer de profissionais e considerar a legitimidade dos saberes e valores que permeiam tais práticas, suas estratégias e táticas próprias. Este é o deslocamento da perspectiva presente em pesquisas do cotidiano que se ocupam das -artes de fazer- dos praticantes, na busca da compreensão de suas regras próprias e de seu desenvolvimento (19).

Em concordância com essa afirmativa, está Maria José Coelho, enfermeira, que em duas pesquisas provenientes de sua tese de doutoramento, descreveu os vários tipos de cuidar e seus produtos baseados no cotidiano de Certeau. É importante destacar que essas pesquisas de reflexão crítica possibilitaram a identificação das maneiras de cuidar em enfermagem juntoà clientela assistida estruturando o sistema de cuidar/cuidados, ou seja, o próprio cliente foi o protagonista da definição dos cuidados, aos quais era submetido. Sendo assim, a reflexão e discussão sobre o conteúdo com os envolvidos no processo de cuidar trouxeram uma contribuição mais ampla e proveitosa para ambos os interessados.

Essa interação entre profissional e cliente e entre profissionais da mesma área ou de áreas diferentes estimula o desenvolvimento da tolerância acadêmica e da ideia de trabalho em equipe inter e multidisciplinar, trazendo mais benefícios para o cliente.

Desse modo, o trabalho interdisciplinar desenvolvido por equipes multiprofissionais em saúde baseia-se em determinantes como: relação interpessoal proveniente do processo de trabalho, cuidado centrado integralmente no cliente e complementação das áreas dos saberes.

Com base nisso, a complementação dos diversos saberes profissionais durante a efetuação da assistência fornece maior 
dinamismo no desenvolvimento do cuidado, minimizando as influências do modelo biologicista, pautado no atendimento multidisciplinar, o que ressignifica dessa forma, a qualidade do atendimento prestado (20).

A partir disso, percebe-se que através da abordagem interdisciplinar, as relações de poder tendem a se horizontalizar frente a um problema característico do processo de trabalho, e a equipe passa a buscar soluções em comum, a partir da troca de saberes, o que gera uma fecunda e mútua aprendizagem.

Diante do exposto, percebe-se que uma equipe multiprofissional possui uma diversidade de informações a oferecer possibilitando uma discussão mais rica e com resultados mais fidedignos com a realidade.

A Teoria das Representações Sociais, por exemplo, tem por objetivo explicar os fenômenos do homem a partir de uma perspectiva coletiva, sem perder de vista a individualidade.

As representações sociais têm como uma de suas finalidades tornar familiar algo não familiar, isto é, um classificar, categorizar e nomear novos acontecimentos e ideias com as quais não tínhamos tido contato anteriormente, possibilitando, assim, a compreensão e manipulação desses novos acontecimentos a partir de ideias, valores e teorias preexistentes e internalizados por nós e amplamente aceitas pela sociedade ${ }^{21}$.

Diante dessas afirmativas, o artigo (4) utilizado discute a interação entre profissional e cliente e afirma que, uma vez caracterizada a experiência e vivida à interação com o usuário, se revela a intersubjetividade dos atores, que provoca, então, a construção de uma realidade social. Essa realidade possibilita a prática de um cuidar mais condizente com as expectativas do cliente.

A pesquisa de reflexão crítica e de representação social possuem uma convergência interessante no aspecto relacionado a exposição de opiniões e busca pelo conhecimento como forma de fortalecer e fundamentar seus pensamentos e significados em relação a determinado tema. Assim como essas metodologias, que constroem suas opiniões através do levantamento de informações e de pensamentos diversos, a abordagem multirreferencial, à medida que pretende assegurar a complexidade de tais fenômenos, pressupõe, para a sua compreensão, a conjugação de uma série de abordagens e disciplinas, levando-nos a um tipo de conhecimento que se caracteriza, principalmente, pela pluralidade e heterogeneidade.

Essa afirmativa corrobora com os preceitos da Teoria Fundamentada nos Dados (TFD) que consiste numa -abordagem de pesquisa qualitativa com o objetivo de descobrir teorias, conceitos e hipóteses, baseados nos dados coletados, ao invés de utilizar aqueles predeterminados" (22:2). Possui raízes no Interacionismo Simbólico (IS) e compreende a realidade a partir do conhecimento da percepção ou significado que certo contexto ou objeto tem para a pessoa. A intenção da TFD é descobrir um modelo conceitual que explique o fenômeno a ser investigado e possibilite ao investigador desenvolver e relacionar conceitos. 
A TFD consiste em método para construção de teoria com base nos dados investigados de determinada realidade, de maneira indutiva ou dedutiva que, mediante a organização em categorias conceituais, possibilita a explicação do fenômeno investigado.

Destaca-se que a TFD não parte de teorias já existentes e, sim, se fundamenta a partir de dados da própria cena social sem a pretensão de refutar ou provar o produto de seus achados, mas, sim, acrescentar outras/novas perspectivas para elucidar o objeto investigado (23). Ou seja, parte da realidade para construir seu relato. Sendo assim, é uma metodologia que se adequa perfeitamente ao tipo de pesquisa relacionada.

Já a revisão sistemática é definida "como uma síntese de estudos primários que contém objetivos, materiais e métodos claramente explicitados e que foi conduzida de acordo com uma metodologia clara e reprodutível" (24).

As revisões sistemáticas reúnem grande quantidade de resultados de pesquisas clínicas, discutindo diferenças entre estudos primários que tratam do mesmo objeto. Os estudos primários são, neste caso, os sujeitos da pesquisa.

Como o nome sugere, tais revisões são sistemáticas na abordagem e usam métodos explícitos e rigorosos para identificar textos, fazer apreciação crítica e sintetizar estudos relevantes.

Esta metodologia fundamenta-se no Mo- produção científica mundial, do crescente número de intervenções, tecnologias, medicamentos e terapias na área de saúde, e da necessidade de validar os resultados obtidos a partir de vários estudos sobre determinada questão, a fim de subsidiar a tomada de decisão, seja clínica, gerencial, política, seja epidemiológica (25).

A pesquisa que utilizou a revisão sistemática tinha como objeto de estudo o cuidado interdisciplinar desenvolvido pelo enfermeiro junto à equipe multiprofissional em saúde e aponta para a escassez de referências que refletem sobre a interdisciplinaridade na dinâmica de atendimento nos serviços de saúde. Demonstra também que o trabalho interdisciplinar desenvolvido por equipes multiprofissionais em saúde é diretamente determinado por relações interpessoais estabelecidas através de interação profissional-profissional e usuário-profissional.

Os trabalhos de pesquisa também referiram a utilização da concepção teórica da complexidade. A ideia de complexo, de complexidade, sempre foi usada, mas só modernamente vem ganhando foros de um novo referencial nas ciências. Por conseguinte, fala-se em teoria da complexidade, paradigma da complexidade, epistemologia da complexidade nas ciências, tecnologias e na política.

Edgar Morin prefere usar a expressão pensamento complexo, que parece ser menos ambiciosa e a que melhor responde às necessidades do setor saúde no momento (26).

Nos estudos realizados por Morin, observa-se que o conhecimento do pensamento 
complexo não se limita simplesmente sobre as análises da ciência, mas também sobre a profundidade do conhecimento que existe nas artes, na literatura, na poesia, entre outras formas (27).

O pensamento complexo não tem como objetivo a ruptura de uma forma de pensar em outra e nem de criar metodologias, e sim métodos que ajudem no desenvolvimento e complementação na forma de pensar $(26,27)$.

Desta forma, o pensamento complexo é um pensamento que trata com a incerteza e que é capaz de conceber a organização. É o pensamento apto a reunir, contextualizar, globalizar, mas ao mesmo tempo, a reconhecer o singular, o individual e o concreto.

Para subsidiar essa reflexão sobre a junção de ideias, pensamentos, atos e atuações, devemos entender que existem cuidados executados de maneiras singulares que corroboram com cada conhecimento-disciplina. $\mathrm{O}$ foco dessa reflexão está na interseção entre esses domínios $(17,28)$.

Um dos efeitos dessa separação, dessa fragmentação foi a distribuição do ensino em disciplinas. A disciplina nada mais é do que um ramo do saber do conhecimento voltado para ele mesmo. Diante dessas dicotomias, o pensamento complexo coloca a atitude de transdisciplinaridade, para produzir diálogos entre os diferentes saberes, permitindo a emersão das transversalidades, ou seja, saindo do referencial paradigmático da dicotomia para o da conexão dos saberes. $(7,29)$

Transdisciplinaridade não significa apenas disciplinas que colocaram entre si em determinados projetos com um conhecimento em comum, mas significa também, que há um modo de pensar organizador que pode atravessar as disciplinas e que pode proporcionar uma unidade ${ }^{26}$.

No entanto, para que haja a transdisciplinaridade e a transversalidade é necessário um pensamento organizador, que Morin chama de pensamento complexo. Se não há um pensamento complexo não pode haver transdisciplinaridade. Em razão disso, o pensamento complexo tem como base, o complexo. A palavra complexo vem do latim complexus que quer dizer aquilo que é tecido em conjunto (30).

Quatro textos do quantitativo total de trabalhos de pesquisa que fazem parte desse artigo definiram a epistemologia da complexidade como concepção metodológica, e como objeto: a existência de uma relação de -não cuidado- de enfermagem em um serviço de emergência hospitalar junto aos sujeitos internados ou em observação; a complexidade em saúde da família e a formação do futuro profissional; As interfaces do cuidado pelo olhar da complexidade; a (in)visibilidade do cuidado e da profissão de enfermagem no espaço de relações. A utilização da epistemologia da complexidade como uma forma de responder a demanda da complexidade dessas pesquisas é pertinente, visto que são inteiramente convergentes, além de todos os objetos estarem atentos às relações entre os profissionais e entre os profissionais e o cliente.

Os demais trabalhos de pesquisa, que utilizam a Reflexão teórica e a Teoria Fundamentada nos Dados como metodologia, versam sobre a aproximação da 
Sistematização da Assistência de Enfermagem com a complexidade de Edgar Morin; O significado do cuidado de enfermagem a partir da ótica do pensamento complexo; e Sistematização da Assistência de Enfermagem à luz do pensamento complexo.

Embora esses trabalhos utilizem concepções metodológicas diferentes, apresentam similaridades em seus desfechos. Eles entendem que cada indivíduo cuidado possui suas especificidades e multidimensionalidade e que cada cuidado executado deve ser interativo, complementar e multiprofissional, que precisa ir além da Enfermagem, ou seja, necessita extrapolar os limites do saber disciplinar e caminhar na perspectiva do trabalho conjunto e complementar (31).

Há a necessidade ainda de que os cuidadores conheçam melhor esta realidade, a fim de que possam unir forma e conteúdo, quantidade e qualidade, razão e sensibilidade. Com isto, acredita-se que se possa cuidar melhor, confrontando e emancipando este cuidado, entendendo dialeticamente a realidade complexa para nela agir e cuidar.

Compete ao profissional de enfermagem, na interação que estabelece com o cliente, propiciar o cuidado uni e multidimensional, segundo o pensamento complexo, buscando contemplar as particularidades e multiplicidades do ser. $\mathrm{O}$ pensamento complexo possibilita uma nova forma de contemplar a realidade que permeia o espaço do cuidar em enfermagem, desvinculando-se do pensamento reducionista, embasado na neutralidade de valores, que contempla o ser humano de forma fragmentada, isolada e unilateral. As- sim, o pensar complexo tem o propósito de unificar, clarificar e contextualizar a relação do ser humano com si próprio, com o outro e com o universo, bem como expressar as singularidades e pluralidades do ser $(29,32)$.

Diante do exposto, percebemos que as concepções metodológicas utilizadas nas pesquisas envolvidas nesse trabalho possuem um cunho questionador, crítico, que busca informações internas e externas ao seu grupo, ao pensamento e a prática. Sendo assim, permite aos profissionais e clientes o contato com um fazer construído por várias mãos e experiências, $o$ que corrobora com a forma de atuar da interdisciplinaridade, a transdisciplinaridade e complexidade.

\section{Conclusão}

A análise do conjunto de dados permitiu concluir que as pesquisas objetivaram compreender o cuidado sob o foco da complexidade de Edgar Morin, da influencia do ambiente de Florence Nightingale, organização da assistência de Enfermagem de Wanda Aguiar Horta, Cuidados de Coelho e Cotidianos de Certeau. Cada um, com sua especificidade, permite ao profissional atender o cliente de uma forma mais adequada.

As concepções teóricas e metodológicas utilizadas nesses trabalhos científicos e suas relações com a prática profissional, com a forma de visualizar a temática, permitiram a extração de ideias e informações fidedignas sobre o cuidado ao indivíduo.

Faz-se necessário destacar, a convergência entre as concepções teóricas e 
metodológicas, fato que possibilitou o exponenciamento dos significados de cada participante das pesquisas sobre $o$ cuidado.

O que deve ser ressaltado deste encontro de saberes é o fato da possibilidade e da necessidade de unificação das propostas de ação junto ao indivíduo que necessita de cuidados. Essa atuação inter e transdisciplinar permitirá um atendimento mais eficaz, humano e contextualizado a pessoa e grupo.

É importante ressaltar que o enfermeiro não executa seu trabalho de forma isola$\mathrm{da}$, tampouco independente dos demais profissionais. Em outras palavras o cuidado é uma atividade de enfermagem e

\section{Referência}

1. Erdmann AL, Marcelino G, Nascimento KC, Ribeiro, JA. As interfaces do cuidado pelo olhar da complexidade: um estudo com um grupo de pós-graduandos de enfermagem. Esc Anna Nery, R. Enferm. 2005 dez; 9(3): 411-20.

2. Boff L. Saber cuidar: ética do humano, compaixão pela terra. Petrópolis (RJ): Vozes; 1999.

3. Nightingale F. Notas sobre enfermagem: o que é e o que não é. Prefácio de leda Barreira e Castro. Tradução de Amália Corrêa de Carvalho. São Paulo (SP): Cortez; 1989.

4. Tanaka LH, Leite MMJ. O cuidar no processo de trabalho do enfermeiro: visão dos professores. Ver Bras Enferm 2007; 60(6):681-6.

5. Vale EG, Pagliuca LMF. Construção de um conceito de cuidado de enfermagem: também uma atividade interdisciplinar, que se relaciona direta ou indiretamente com outro profissional. Desse modo o resultado da atuação da equipe passa a ter características interdisciplinares e transdisciplinares, sem perder a individualidade e o domínio de cada profissional.

Desta forma, faz-se necessário pensar e repensar as estratégias utilizadas por cada profissional no desenvolvimento de suas atividades, inserindo o contexto da complexidade como algo imperativo para o desenvolvimento de uma prática de cuidado de qualidade e, consequentemente, de extrema importância para a saúde do indivíduo. Essa mudança certamente estimulará reflexões que futuramente poderão trazer outras conquistas.

contribuição para o ensino de graduação. Rev Bras Enferm, Brasília, 2011. Jan-fev; 64 (1): 106-13.

6. Mazon L, Trevizan MA. Fecundando o processo da interdisciplinaridade na iniciação científica. Ver Latino-americana Enfermagem. 2001; 9(4):83-7.

7. Teixeira, ER (Org.). Psicossomática no Cuidado em Saúde - Atitude Transdisciplinar. São Paulo, Yendis, 2009.

8.Collière MF. Promover a vida: da prática das mulheres de virtude aos cuidados de enfermagem. Lisboa (PT): Lidel; 1999.

9. Terra MG, Camponogara S, Silva LC, Girondi JBR, Nascimento K, Radunz V, Santos EKA. O significado de cuidar no contexto do pensamento complexo: novas possibilidades para a enfermagem. Texto 
10. Lopes FL, Szewczyk MSC, Lunardi VL, Santos SSC. SAE como um novo fazer na atividade cuidativa da enfermeira com base na complexidade de Edgar Morin. Cogitare Enferm 2007 jan/mar; 12(1):109-13

11. Oguisso T. Trajetória histórica e legal da enfermagem. Editora Manole, 2005.

12. Carvalho V. Cuidando, pesquisando e ensinando: acerca de significados e implicações da prática da enfermagem. Rev Latino-am Enfermagem 2004 set/out; 12(5):806-15

13. Carvalho V. Por uma epistemologia do cuidado de Enfermagem e a formação dos sujeitos do conhecimento na área da Enfermagem - do ângulo de uma visão filosófica. Esc Anna Nery Enferm, 2009 abr/jun; 13(2):406-14.

14. Galvão CM, Sawada NO, Trevizan MA. Revisão sistemática: recurso que proporciona a incorporação das evidências na prática da enfermagem. Rev Latino-am Enfermagem 2004 Mai-Jun; 12(3):549-56

15. Ardoino, J. Abordagem multirreferencial (plural) das situações educativas e formativas. In: Barbosa JG. Multirreferencialidade nas ciências e na educação (pp. 24-41). São Carlos: Editora da UFSCar. 1998.

16. HORTA WA. Processo de Enfermagem. EPU, 1979.

17. Coelho MJ. Produtos dos cuidados de enfermagem. Rev Bras Enferm. 2009; 62(6):919-22.

18. Duran MCG. Maneiras de pensar o cotidiano com Michel de Certeau. Diálogo Educ., Curitiba, v. 7, n. 22, p. 115-128, set./dez. 2007.

19. Certeau, M. de. Ainvenção do cotidiano: 1, Artes de fazer. Petrópolis: Vozes, 1994.
20. Silva PS, Felippe KC. O cuidado interdisciplinar do enfermeiro na equipe multiprofissional em saúde: revisão sistemática. R. pesq. Cuid. Fundam. Online 2011. Out. / dez. 3(4):2569-78.

21. Moscovici, S. Representações Sociais: Investigações em Psicologia Social / Serge Moscovici: editado em inglês por Gerard Duveen: traduzido por Pedrinho A. Guareschi. 6. ed. - Petrópolis, RJ: Vozes, 2009.

22. Dantas CC, Leite JL, Lima SBS, Stipp MAC. Teoria fundamentada nos dadosaspectos conceituais e operacionais: metodologia possível de ser aplicada na pesquisa em enfermagem. Rev Latino-am Enfermagem 2009 julho-agosto; 17(4).

23. Strauss A, Corbin J. Pesquisa Qualitativa: Técnica e procedimentos para o desenvolvimento da teoria fundamentada. $2^{\mathrm{a} e d}$. Porto Alegre: Artmed; 2008.

24. Lopes ALM, Fracolli LA. Revisão sistemática de literatura e metassíntese qualitativa: considerações sobre sua aplicação na pesquisa em enfermagem. Texto Contexto Enferm, Florianópolis, 2008 Out-Dez; 17(4): 771-8.

25. Cullum N, Ciliska D, Haynes RB, Marks S. Enfermagem Baseada em Evidências: uma introdução. Tradução Ana Thorell. Porto Alegre: Artmed, 2010.

26. Morin E, Louis Le moigne J. Os setes saberes Necessários à educação do Futuro. Ed. Cortez, 2003.

27. Ferreira CL, Gallo H. Pensamento complexo: Reflexões sobre a vida contemporânea. Complexus -Instituto Superior de Engenharia, Arquitetura e DesignCEUNSP, Salto-SP, ano. 1, n.1, p. 01-22, março de 2010. <Disponível em: www. engenho.info>

28. Coelho MJ. Maneiras de cuidar em Enfermagem. Rev Bras Enferm. 2006; 59 (6):745-51. 
29. Morin E. Introdução ao pensamento complexo. Porto Alegre (RS): Sulina; 2006.

30. Moretti-Pires RO. Complexidade em saúde da família e a formação do futuro profissional de saúde. Interface Comunicação Saúde Educação. Jul/set 2009; 13(30): 153-66.

31. Nascimento KC, Backes DS, Koerich MS. Sistematização da assistência de en- fermagem: vislumbrando um cuidado interativo, complementar e multiprofissional. Rev Esc Enferm USP, 2008; 42(4): 643-8.

32. Baggio MA, Callegaro GD, Erdmann AL. Relações de -Não Cuidado- de enfermagem em uma emergência: que cuidado é esse? Esc. Anna Nery (impr.) 2011; 15(1):116-23. 
